\title{
Un « trésor infini de beauté originale »: le premier recueil de chants populaires roumains
}

\section{Ana-Maria Gîrleanu-Guichard}

\section{Q OpenEdition}

\section{Journals}

Édition électronique

URL : http://journals.openedition.org/rbnu/713

DOI : $10.4000 /$ rbnu. 713

ISSN : 2679-6104

Éditeur

Bibliothèque nationale et universitaire de Strasbourg

\section{Édition imprimée}

Date de publication : 1 novembre 2017

Pagination : 100-103

ISBN : 9782859230678

ISSN : 2109-2761

\section{Référence électronique}

Ana-Maria Gîrleanu-Guichard, « Un « trésor infini de beauté originale » : le premier recueil de chants populaires roumains », La Revue de la BNU [En ligne], 16 | 2017, mis en ligne le 01 juillet 2019, consulté le 15 décembre 2020. URL : http://journals.openedition.org/rbnu/713 ; DOI : https://doi.org/10.4000/ rbnu.713

\section{(c) (1) ()}

La Revue de la BNU est mise à disposition selon les termes de la Licence Creative Commons Attribution - Pas d'Utilisation Commerciale - Partage dans les Mêmes Conditions 4.0 International. 


\section{BALLADES}

E T

\section{CHANTS POPULAIRES \\ DE LA ROUMANIE}

[PRINGIFAUTES DRIUBIENAES]

RECUEILLIS ET TRADUITS

PAR V. ALEXANDRI

$\triangle$ VBG UNB INTRODUCTION

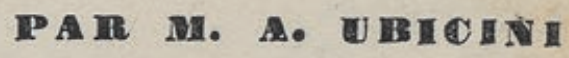

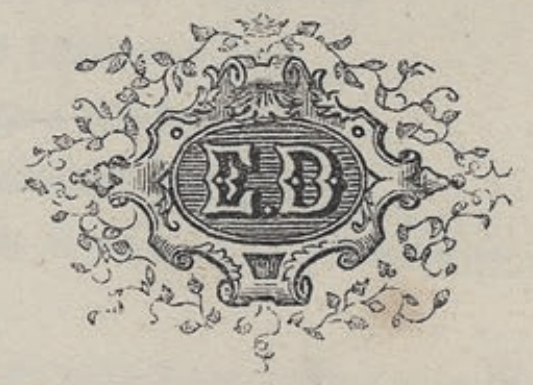

\section{PAR IS}

E. DENTU, LIBRAIRE-ÉDITEUR

PALAIS-ROYAL, 13 GALERIF VITRÉE

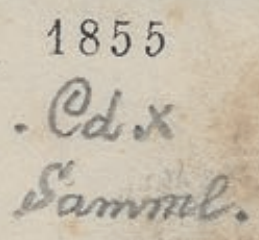


ROUMANIE

\section{UN «TRÉSOR INFINI DE BEAUTÉ ORIGINALE » : LE PREMIER RECUEIL DE CHANTS POPULAIRES ROUMAINS}

PAR ANA-MARIA GÎRLEANU-GUICHARD

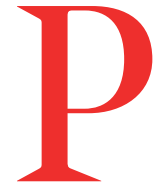

armi les documents rares relatifs à la culture roumaine qu'abritent les riches collections de la BNU se trouve un recueil de poèmes populaires paru en 1855, à Paris. Son ex-libris nous apprend qu'il a appartenu à Auguste Schneegans (1835-1898), homme politique et journaliste alsacien qui avait voyagé pendant sa jeunesse dans les principautés roumaines.

Ce qui se joue dès le titre de l'ouvrage, Ballades et chants populaires de la Roumanie (principautés danubiennes), c'est l'usage même du nom désignant le territoire de ce "peuple naturellement poète " (A. Ubicini) : la "Roumanie », que la précision "principautés danubiennes " aide le lecteur à situer sur la carte, quelque part aux confins orientaux du continent, près de l'embouchure du Danube.

À cette époque, politiquement, la Roumanie n'existe pas encore. Le gentilé même de « Roumains » commence à peine à entrer dans le langage courant en français, remplaçant peu à peu les termes «Valaques », "Moldaves » ou "Moldo-Valaques » auparavant en usage $^{1}$. Depuis des siècles, les Roumains vivent séparés en trois pays : la Valachie et la Moldavie, vassales du sultan turc depuis le $15^{\mathrm{e}}$ siècle, et la Transylvanie conquise par les Habsbourg à la fin du $17^{\mathrm{e}}$ siècle $^{2}$. Tout comme l'unification politique italienne ou allemande, l'unification roumaine sera un processus de longue durée, commencé en 1859 par l'union des «principautés danubiennes » et achevé en 1918, par l'intégration de la Transylvanie au royaume de Roumanie.

Quel est donc cet ouvrage au titre visionnaire? Qui en est l'artisan ? Dans quel contexte historique paraît-il et quelle en est la signification ?

Fruit d'une dizaine d'années de travail, le premier recueil de poèmes populaires roumains est publié à Jassy, en deux tomes parus en 1852 et 1853, par Vasile Alecsandri (1821-1890). Écrivain, folkloriste, homme politique et diplomate, Alecsandri fait partie des intellectuels issus des élites autochtones qui contribuent à la réforme démocratique et à la fondation de la Roumanie moderne, sur le modèle occidental. Ils sont connus dans l'historiographie roumaine comme les « quarante-huitards ", pour avoir introduit dans les pays roumains les idées libérales de la révolution française de $1848^{3}$. Nombre de ces jeunes gens avaient 
suivi au Collège de France les cours de Jules Michelet et d'Edgar Quinet, avec lesquels ils avaient noué des liens durables.

Formé à Paris dans ces cercles, Alecsandri relève très tôt, dans sa correspondance avec Nicolae Bălcescu et Alecu Russo ${ }^{4}$, la nécessité d'un recueil de chants populaires roumains et commence à se consacrer avec dévouement à cette tâche. Il sillonne la Moldavie, s'arrêtant aux fêtes paysannes dans les villages, voyageant dans les montagnes pour écouter chanter les bergers. Il recueille ainsi tout chant " ancien " et « beau » et sollicite l'aide d'autres collaborateurs, en particulier d'Alecu Russo, pour réunir le plus grand nombre de poèmes de toutes les contrées roumaines.

Comme les romantiques de son temps, Alecsandri est persuadé que la poésie populaire exprime la voix et le "génie d'un peuple » (Herder, Michelet). À cette « époque où les peuples s'éveillent au soleil de la civilisation et de la liberté ", le peuple roumain mérite d'être connu aussi pour le « trésor infini de beauté originale » qu'est sa poésie populaire, laquelle reflète bien «toutes les dispositions de son cœur ainsi que la splendeur de son génie ", note Alecsandri dans ses études consacrées à la littérature populaire ${ }^{5}$. Il retravaille longuement, amoureusement, son « tas de manuscrits » (« Je suis tombé amoureux de la poésie populaire comme d'une jeune fille des Carpathes ", avoue-t-il) pour en tirer deux volumes de ballades, réédités en 1866, enrichis de "doïnas " (poèmes lyriques) et de «horas" (chants scandés), sous le titre Poezii popolare ale Românilor (Poèmes populaires des Roumains, 1866).

Le titre de cette dernière édition dévoile l'enjeu majeur de la publication : montrer que les Roumains des trois provinces historiques partagent la même langue, la même sensibilité littéraire et des traditions et coutumes semblables, dont certaines remontent au temps des Romains. Saluée dans les revues roumaines comme une "révolution ", la parution de ce recueil constitue un événement à une époque où la langue littéraire roumaine cherche sa voie entre imitations et traductions (en particulier des romantiques européens : Lamartine, Byron, Schiller). L'anthologie de ballades de 1852 allait également fournir la matière pour des recueils de poèmes traduits en français, anglais, allemand, de 1855 à $1857^{6}$, et diffusés à travers l'Europe dans un moment décisif pour le sort des principautés roumaines ${ }^{7}$.

Le volume paru chez Edouard Dentu en 1855 est précédé par une vaste introduction d'A. Ubicini, l'un des défenseurs de la cause roumaine au plan européen ${ }^{8}$. Son introduction se veut un aperçu de l'histoire du peuple roumain et de sa langue, insistant sur leur " noble ascendance ", qu'il s'agit de rappeler aussi bien aux Roumains qu'à la « savante Europe ". Il entreprend de relever ce qui dans la culture et la langue roumaine "fait souvenir de l'ancienne Rome ", véhiculant ainsi nombre d'idées communes aux discours des écrivains roumains (Alecsandri, Bălcescu) et français (en particulier Michelet et Quinet ${ }^{9}$ ) engagés dans la reconnaissance de la « communauté de race et d'intérêts " qui relie les nations de l'Occident au sort des principautés danubiennes et dont le véritable enjeu est le «maintien de l'équilibre en Europe » (Ubicini).

Parmi ces " chants " anonymes, il convient de mentionner deux poèmes emblématiques : La Petite brebis (Mioriţa) et Le Monastère d'Argeş dont les riches interprétations allaient nourrir en Roumanie pendant près d'un siècle le débat sur la «spécificité nationale ». Liée à la construction légendaire d'un des édifices monastiques roumains les plus connus, la ballade du Monastère d'Argeş est fondée sur le mythe du sacrifice pour la création, décliné sous diverses formes dans l'espace culturel du sud-est européen. Le maître maçon Manole et ses compagnons doivent bâtir « un beau monastère / sans pareil au monde ". Mais ce que les maçons bâtissent le jour s'écroulera la nuit tant qu'un être humain ne sera pas sacrifié dans les fondations. Victime prédestinée au sacrifice par son amour, Ana, la femme du maître maçon, sera emmurée pour que l'édifice demeure.

Placée en ouverture du recueil, la ballade de La Petite brebis est fondée sur un noyau narratif simple: un jeune berger est averti, par une agnelle voyante, de sa mort imminente. Au lieu de riposter, il envisage sa disparition comme des noces cosmiques avec « la fiancée du monde ", la Mort, et charge la brebis de ses dernières volontés. La nature entière est transfigurée au cours de ce rituel de passage (« le soleil avec la lune ont tenu la couronne sur ma tête, j'ai eu pour témoins les pins et les platanes des forêts, pour prêtres les hautes montagnes, pour orchestre les oiseaux, des 
milliers d'oiseaux, et pour flambeaux les étoiles du firmament »). Michelet, qui avait donné la première traduction française de cette ballade en 1854 dans les Légendes démocratiques $d u$ Nord, la tenait pour « un chant du caractère le plus antique, une chose sainte et touchante à fendre le cœur ${ }^{10}$.

Le volume est bien reçu dans les milieux intellectuels libéraux. Prosper Mérimée relève, dans un article du Moniteur universel, la valeur artistique de ces créations qu'il rapproche des poèmes populaires grecs traduits par Claude Fauriel, ainsi que des poèmes serbes. D'autres chroniques favorables paraissent dans la Revue des deux mondes. "Ces poèmes ont attiré l'attention des gens érudits et éveillé leur sympathie pour notre nation $[. . .]^{11}$ ", écrit avec satisfaction Alecsandri dans une lettre adressée à la princesse Elena Cuza.

Au même titre que les conférences publiques, les missions diplomatiques, les publications consacrées à la langue et à la culture roumaines, la parution des poèmes populaires roumains en France, au moment de «l'éveil des nations " européennes, s'inscrit dans un ample programme politique mis en œuvre par les « quarante-huitards " roumains, programme dont l'essentiel pourrait s'énoncer dans les termes mêmes d'Alecsandri, prononcés lors d'une conférence tenue à Paris en 1848 : « montrer qu'il existe au monde une Roumanie, même si elle ne figure pas dans les livres de géographie ${ }^{12}$. Et parvenir à l'inscrire sur la carte de l'Europe.

\section{NOTES}

1- Appelés « Valaques » par les historiens étrangers, les Roumains se disent eux-mêmes « Roumains » bien avant l'émergence de l'État roumain moderne au $19^{\mathrm{e}}$ siècle, ainsi que l'attestent, dès le $16^{\mathrm{e}}$ siècle, plusieurs sources écrites occidentales.

2- Ces trois pays historiques n'ont connu qu'une union éphémère (1600-1601) sous le règne de Michel le Brave.

3- Comme d'autres pays européens, les trois provinces roumaines ont connu des mouvements révolutionnaires en 1848, suite aux événements de Paris : la « révolution des poètes » en Moldavie, à laquelle a participé Alecsandri, une insurrection sanglante en Valachie et en Transylvanie. Ces mouvements ont échoué, mais ils ont introduit des idées mises en pratique plus tard, au fil du $19^{\mathrm{e}}$ siècle.

4- Nicolae Bălcescu (1819-1852), historien et écrivain politique, et Alecu Russo (1819-1859), poète et homme politique, sont deux figures de proue de la «génération de 1848 ».

5- V. Alecsandri, « Românii şi poezia lor » (1849-1850), in Proză, I, Bucarest, Editura Minerva, 1994, p. 104-144 ; «Poezia poporală », in Poezii populare ale Românilor, Gh. Vrabie (éd.), Bucarest, Editura pentru literatură, 1965, p. 99-100. Les traductions du roumain nous appartiennent.

6- Voir par exemple Henry Stanley, Fleurs de Roumanie / Rouman Anthology or Selections of Rouman Poetry, Ancient and Moderne, Being a Collection of National Ballads of Moldavia and Wallachia, Hertford, 1856. W. von Kotzebue a traduit et publié à Berlin un recueil de poèmes populaires roumains : Rumänische Volkspoesie. Gesammelt und geordnet von B. Alecsandri, Berlin, 1857. Lié aux milieux roumains, il est devenu membre de l'Académie roumaine en 1884 .

7- En effet, les grandes puissances européennes venaient de garantir, par le traité de Paris de 1856 (qui mettait un terme à la guerre de Crimée), l'autonomie de la Moldavie et de la Valachie, tout en les laissant vassales de la Sublime Porte, créant ainsi un contexte favorable à leur union en 1859, suite à l'élection dans les deux États du même prince souverain.

8- Considéré par Alecsandri comme l'un des «nobles champions » défenseurs de la cause roumaine, Abdolonyme Ubicini (1818-1884) est un homme de lettres et historien français d'origine italienne, préoccupé par la « question de l'Orient » qu'il entend porter devant l'Europe. Lié aux « quarante-huitards », il participe à la révolution roumaine de 1848 et devient secrétaire du gouvernement provisoire de Bucarest. Voir A. Ubicini, La Question d'Orient devant l'Europe. Documents officiels, manifestes, notes, firmans, circulaires, etc., depuis l'origine du différend. Annotés et précédés d'une exposition de la question des lieux-saints, Paris, E. Dentu, 1854.

9- Quinet, Edgard, «Les Roumains », in Revue des deux mondes, $2^{\mathrm{e}}$ période, tome 1, 1856 ; Michelet, Jules, « Principautés danubiennes ", in Légendes démocratiques du Nord (1854), Calmann Lévy, 1877 , p. 275 sq.

10- J. Michelet, op. cit., p. 338

11- V. Alecsandri, lettre à la princesse Elena Cuza citée par Gh. Vrabie dans son Introduction aux Poezii populare ale Românilor, éd. cit., p. 22.

12- V. Alecsandri, « Românii şi poezia lor », éd. cit., p. 139 